VOL. 45 (1992) [297-303]

\title{
OSCILLATORY PROPERTIES OF SOLUTIONS OF CERTAIN ELLIPTIC EQUATIONS
}

\section{NORIO YOSHIDA}

Certain elliptic equations of higher order are studied and a sufficient condition is given that every solution is oscillatory in an exterior domain. The principal tool is an averaging technique which enables one to reduce the $\boldsymbol{n}$-dimensional problem to a one-dimensional problem.

Oscillation theory for higher order elliptic equations of the form $\Delta^{m} u+a_{1} \Delta^{m-1} u+$ $\cdots+a_{m} u=0$ ( $\Delta$ is the Laplacian in $\mathbb{R}^{n}$ ) has been investigated by numerous authors. We refer the reader to $[1,4]$ for $n=3$, and to $[3,9]$ for $n \geqslant 2$. In the case where $n=3$, Górowski [5] obtained the oscillation results for the $m$ th metaelliptic equation $\widetilde{L}^{m} u+a_{1} \widetilde{L}^{m-1} u+\cdots+a_{m} u=0$, where $\widetilde{L}=\sum_{j, k=1}^{3} a_{j k}\left(\partial^{2} / \partial x_{j} \partial x_{k}\right)\left(a_{j k}=\right.$ constant $)$.

We are concerned with the oscillatory behaviour of solutions of the elliptic equation

$$
\left(L^{m}+a_{1} L^{m-1}+\cdots+a_{m-1} L+a_{m}\right) u(x)=0, \quad x \in \Omega
$$

where $\Omega$ is an exterior domain of $\mathbb{R}^{n}(n \geqslant 2)$, that is $\Omega$ contains the complement of some $n$-ball in $\mathbb{R}^{n}$. As usual, $x=\left(x_{1}, x_{2}, \ldots, x_{n}\right)$ denotes a point of $\mathbb{R}^{n}$. It is assumed that the coefficients $a_{j}(j=1,2, \ldots, m)$ are real constants, $L$ is the linear elliptic operator with constant coefficients

$$
L=\sum_{j, k=1}^{n} a_{j k} \frac{\partial^{2}}{\partial x_{j} \partial x_{k}}
$$

where $a_{j k}=a_{k j}$ and $\left(a_{j k}\right)$ is positive definite, and $L^{k}$ is the $k$ th iterate of $L$ $(k=1,2, \ldots, m)$.

The purpose of this paper is to present sufficient conditions for all solutions of (1) to be oscillatory in $\Omega$. Our method is an adaptation of that used in [3].

Definition: A function $u: \Omega \rightarrow \mathbb{R}^{1}$ is said to be oscillatory in $\Omega$ if $u$ has a zero in $\{x \in \Omega:|x|>r\}$ for any $r>0$, where $|x|$ denotes the Euclidean length of $x$.

Received 12 May 1991

Copyright Clearance Centre, Inc. Serial-fee code: 0004-9729/92 $\$ A 2.00+0.00$. 
Since $\Omega$ is an exterior domain in $\mathbb{R}^{n}, \Omega$ contains $\mathbb{R}^{n}\left(r_{0}\right) \equiv\left\{x \in \mathbb{R}^{n}:|x|>r_{0}\right\}$ for some $r_{0}>0$. Let $x_{0}=\left(x_{1}^{0}, x_{2}^{0}, \ldots, x_{n}^{0}\right)$ be a fixed point of $\mathbb{R}^{n}\left(r_{0}\right)$ and let $\rho(x)$ be defined by

$$
\rho(x)=\left(\sum_{j, k=1}^{n} A_{j k}\left(x_{j}-x_{j}^{0}\right)\left(x_{k}-x_{k}^{0}\right)\right)^{1 / 2}
$$

where $\left(A_{j k}\right)$ denotes the inverse matrix of $\left(a_{j k}\right)$. There is an $r_{1}>0$ such that $\{x \in$ $\left.\mathbb{R}^{n}: \rho(x)>r_{1}\right\} \subset \mathbb{R}^{n}\left(r_{0}\right)$. Associated with every function $u \in C(\Omega)$, we define the function $M[u](r)$ by

$$
M[u](r)=\frac{1}{\sigma_{n} r^{n-1}} \int_{S_{r}} u \frac{d \sigma}{|\nabla \rho|}, \quad r>r_{1}
$$

where $\sigma_{n}$ denotes the surface area of the unit sphere in $\mathbb{R}^{n}$ and $S_{r}=\left\{x \in \mathbb{R}^{n}: \rho(x)=\right.$ $\boldsymbol{r}\}$.

LEMMA 1. If $u \in C^{2}(\Omega)$, then we obtain

$$
\frac{1}{\sigma_{n} r^{n-1}} \int_{S_{r}} L u \frac{d \sigma}{|\nabla \rho|}=r^{1-n} \frac{d}{d r}\left(r^{n-1} \frac{d}{d r} M[u](r)\right), \quad r>r_{1},
$$

where $L$ is given by (2).

Proof: It is easy to see that ${ }^{t}(\nabla \rho)\left(A_{j k}\right)(\nabla \rho)=1$. Hence, the conclusion follows from a result of Suleimanov [8] (see also [12, Lemma 2.1]).

LEMMA 2. If $u \in C^{4}(\Omega)$, then $M[u](r)$ satisfies

(4) $\frac{1}{\sigma_{n} r^{n-1}} \int_{S_{r}} L^{2} u \frac{d \sigma}{|\nabla \rho|}=r^{1-n} \frac{d}{d r}\left(r^{n-1} \frac{d}{d r}\left(r^{1-n} \frac{d}{d r}\left(r^{n-1} \frac{d}{d r} M[u](r)\right)\right)\right), r>r_{1}$.

Proof: Lemma 1 implies that

$$
\begin{gathered}
M[L u](r)=r^{1-n} \frac{d}{d r}\left(r^{n-1} \frac{d}{d r} M[u](r)\right) \\
\frac{1}{\sigma_{n} r^{n-1}} \int_{S_{r}} L^{2} u \frac{d \sigma}{|\nabla \rho|}=r^{1-n} \frac{d}{d r}\left(r^{n-1} \frac{d}{d r} M[L u](r)\right)
\end{gathered}
$$

Combining (5) with (6) yields the desired identity (4).

THEOREM. Assume that the algebraic equation

$$
z^{m}+a_{1} z^{m-1}+a_{2} z^{m-2}+\cdots+a_{m}=0
$$


has simple roots only and has no real nonnegative root. Then every solution $u \in C^{2 m}(\Omega)$ of (1) is oscillatory in $\Omega$.

Proof: Suppose to the contrary that there exists a solution $u \in C^{2 m}(\Omega)$ of (1) which does not oscillate in $\Omega$. Without loss of generality we may assume that $u>0$ in $\mathbb{R}^{n}\left(r_{2}\right)$ for some $r_{2} \geqslant r_{1}$. The hypothesis implies that

$$
z^{m}+a_{1} z^{m-1}+a_{2} z^{m-2}+\cdots+a_{m}=\prod_{k=1}^{p}\left(z^{2}+2 b_{k} z+\left(b_{k}^{2}+c_{k}^{2}\right)\right) \prod_{k=2 p+1}^{m}\left(z+d_{k}^{2}\right),
$$

where $c_{k}>0(k=1,2, \ldots, p), d_{k}>0(k=2 p+1,2 p+2, \ldots, m),-b_{j} \pm i c_{j} \neq-b_{k} \pm$ $i c_{k}(j \neq k, i=\sqrt{-1})$ and $d_{j} \neq d_{k}(j \neq k)$. Hence, (1) can be written in the form

$$
\left(\prod_{k=1}^{p}\left(L^{2}+2 b_{k} L+\left(b_{k}^{2}+c_{k}^{2}\right)\right) \prod_{k=2 p+1}^{m}\left(L+d_{k}^{2}\right)\right) u=0 .
$$

It follows from a result of Wachnicki [10, Theorem 2] that there exists a unique system $\tilde{u}_{k}(x)(k=1,2, \ldots, p), u_{k}(x)(k=2 p+1,2 p+2, \ldots, m)$ such that

$$
\begin{gathered}
\left(L^{2}+2 b_{k} L+\left(b_{k}^{2}+c_{k}^{2}\right)\right) \tilde{u}_{k}(x)=0 \quad(k=1,2, \ldots, p) \\
\left(L+d_{k}^{2}\right) u_{k}(x)=0 \quad(k=2 p+1,2 p+2, \ldots, m)
\end{gathered}
$$

and

$$
u(x)=\sum_{k=1}^{p} \tilde{u}_{k}(x)+\sum_{k=2 p+1}^{m} u_{k}(x)
$$

(see [4, Lemma 4]). Then we easily obtain

$$
M[u](r)=\sum_{k=1}^{p} M\left[\tilde{u}_{k}\right](r)+\sum_{k=2 p+1}^{m} M\left[u_{k}\right](r)
$$

and we observe, using Lemmas 1 and 2, that

$$
\begin{aligned}
& r^{1-n} \frac{d}{d r}\left(r^{n-1} \frac{d}{d r} M\left[u_{k}\right](r)\right)+d_{k}^{2} M\left[u_{k}\right](r)=0 \\
& r^{1-n} \frac{d}{d r}\left(r^{n-1} \frac{d}{d r}\left(r^{1-n} \frac{d}{d r}\left(r^{n-1} \frac{d}{d r} M\left[\tilde{u}_{k}\right](r)\right)\right)\right) \\
& \quad+2 b_{k} r^{1-n} \frac{d}{d r}\left(r^{n-1} \frac{d}{d r} M\left[\tilde{u}_{k}\right](r)\right)+\left(b_{k}^{2}+c_{k}^{2}\right) M\left[\tilde{u}_{k}\right](r)=0
\end{aligned}
$$


Using the same arguments as in $[3$, p.231], we see that

(11) $r^{(n-1) / 2} M\left[u_{k}\right](r) \approx A_{k} \sin \left(\int_{r}^{r}\left(d_{k}^{2}-\left(1-n^{2}\right) 4^{-1} s^{-2}\right)^{1 / 2} d s+\theta_{k}\right) \quad(r \rightarrow \infty)$

for some constants $A_{k}$ and $\theta_{k}(k=2 p+1,2 p+2, \ldots, m)$. The following system

$$
y^{\prime}=(A+V(r)) y, \quad y={ }^{t}\left(y_{1}, y_{2}, y_{3}, y_{4}\right)
$$

is associated with (10), where

$$
\begin{gathered}
A=\left(\begin{array}{cccc}
0 & 1 & 0 & 0 \\
0 & 0 & 1 & 0 \\
0 & 0 & 0 & 1 \\
-\left(b_{k}^{2}+c_{k}^{2}\right) & 0 & -2 b_{k} & 0
\end{array}\right), \\
V(r)=\left(\begin{array}{cccc}
0 & 0 & 0 & 0 \\
0 & 0 & 0 & 0 \\
0 & 0 & 0 & 0 \\
0 & -\left(2 b_{k} \frac{n-1}{r}-\frac{(n-1)(n-3)}{r^{3}}\right) & -\frac{(n-1)(n-3)}{r^{2}} & -\frac{2(n-1)}{r}
\end{array}\right) .
\end{gathered}
$$

Since $\operatorname{det}(A-\lambda I)=\lambda^{4}+2 b_{k} \lambda^{2}+\left(b_{k}^{2}+c_{k}^{2}\right)$, we find that the characteristic roots of $A$ are $\pm \mu_{1} \pm i \mu_{2}$, where $\mu_{1}=2^{-1 / 2}\left(-b_{k}+\left(b_{k}^{2}+c_{k}^{2}\right)^{1 / 2}\right)^{1 / 2}$ and $\mu_{2}=$ $2^{-1 / 2}\left(b_{k}+\left(b_{k}^{2}+c_{k}^{2}\right)^{1 / 2}\right)^{1 / 2}$. It is easily seen that the characteristic polynomial for $A+V(r)$ is given by

$$
\begin{aligned}
\lambda^{4} & +\frac{2(n-1)}{r} \lambda^{3}+\left(2 b_{k}+\frac{(n-1)(n-3)}{r^{2}}\right) \lambda^{2} \\
& +\left(2 b_{k} \frac{n-1}{r}-\frac{(n-1)(n-3)}{r^{3}}\right) \lambda+b_{k}^{2}+c_{k}^{2} .
\end{aligned}
$$

Using Ferrari's formula (see $\left[11\right.$, p.190]), we conclude that the characteristic roots $\lambda_{j}(r)$ of $A+V(r)$ can be written in the form

$$
\begin{array}{ll}
\lambda_{j}(r)=-\frac{n-1}{2 r}+\mu_{1}(r)+(-1)^{j+1} i \mu_{2}(r) & (j=1,2), \\
\lambda_{j}(r)=-\frac{n-1}{2 r}-\mu_{1}(r)+(-1)^{j+1} i \mu_{2}(r) & (j=3,4),
\end{array}
$$

where $\lim _{r \rightarrow \infty} \mu_{k}(r)=\mu_{k}(k=1,2)$. We easily see that

$$
\int_{r_{1}}^{\infty}\left|V^{\prime}(r)\right| d r<\infty \quad \text { and } \quad \lim _{r \rightarrow \infty} V(r)=0
$$


Then there are the solutions $\Phi_{j}(r)(j=1,2,3,4)$ of $(12)$ and $\tilde{r}\left(r_{1} \leqslant \tilde{r}<\infty\right)$ such that

$$
\lim _{r \rightarrow \infty} \Phi_{j}(r) \exp \left(-\int_{\tilde{r}}^{r} \lambda_{j}(s) d s\right)=p_{j} . \quad(j=1,2,3,4),
$$

where each $p_{j}(j=1,2,3,4)$ is a characteristic vector of $A$ associated with $\mu_{1}+$ $(-1)^{j+1} i \mu_{2}(j=1,2),-\mu_{1}+(-1)^{j+1} i \mu_{2}(j=3,4)$ (see [2, p.92]). Hence, the following holds:

$$
\begin{gathered}
\Phi_{j} r^{(n-1) / 2} \approx P_{j} \exp \left(\int_{\tilde{r}}^{r} \mu_{1}(s) d s\right)\left(\cos \int_{r}^{r} \mu_{2}(s) d s+(-1)^{j+1} i \sin \int_{\tilde{r}}^{r} \mu_{2}(s) d s\right) \\
(r \rightarrow \infty ; j=1,2), \\
\Phi_{j} r^{(n-1) / 2} \approx P_{j} \exp \left(-\int_{r}^{r} \mu_{i}(s) d s\right)\left(\cos \int_{r}^{r} \mu_{2}(s) d s+(-1)^{j+1} i \sin \int_{r}^{r} \mu_{2}(s) d s\right) \\
(r \rightarrow \infty ; j=3,4),
\end{gathered}
$$

where $P_{j}=K_{j} p_{j}$ for some constants $K_{j} \in \mathbb{R}^{1}(j=1,2,3,4)$. Since $M\left[\tilde{u}_{k}\right](r)$ is a realvalued function and a linear combination of the first components of $\Phi_{j}(j=1,2,3,4)$, we obtain

$$
\begin{aligned}
r^{(n-1) / 2} M\left[\widetilde{u}_{k}\right](r) \approx & B_{k} \exp \left(\int_{\tilde{\tau}}^{r} \mu_{1}(s) d s\right) \sin \left(\int_{\tilde{\tau}}^{r} \mu_{2}(s) d s+\sigma_{k}\right) \\
& +C_{k} \exp \left(-\int_{\tilde{\tau}}^{r} \mu_{1}(s) d s\right) \sin \left(\int_{\tilde{\tau}}^{r} \mu_{2}(s) d s+\tau_{k}\right) \quad(r \rightarrow \infty)
\end{aligned}
$$

for some constants $B_{k}, C_{k}, \sigma_{k}$ and $\tau_{k}(k=1,2, \ldots, p)$. Combining (9), (11) and (13) yields

$$
\begin{aligned}
r^{(n-1) / 2} M[u](r) \approx & \sum_{k=2 p+1}^{m} A_{k} \sin \left(\int_{\tilde{r}}^{r}\left(d_{k}^{2}-\left(1-n^{2}\right) 4^{-1} s^{-2}\right)^{1 / 2} d s+\theta_{k}\right) \\
& +\sum_{k=1}^{p} B_{k} \exp \left(\int_{r}^{r} \mu_{1}(s) d s\right) \sin \left(\int_{\tilde{r}}^{r} \mu_{2}(s) d s+\sigma_{k}\right) \\
& +\sum_{k=1}^{p} C_{k} \exp \left(-\int_{r}^{r} \mu_{1}(s) d s\right) \sin \left(\int_{r}^{r} \mu_{2}(s) d s+\tau_{k}\right) \quad(r \rightarrow \infty) .
\end{aligned}
$$

Since $u>0$ in $\mathbb{R}^{n}\left(r_{2}\right)$, the left hand side of (14) is positive for $r>r_{2}$. However, the right hand side of (14) changes sign in an arbitrary interval $(r, \infty)$ (see $[4$, Lemma 6]). 
This is a contradiction. If (7) has only simple negative or only simple complex roots, then we replace (8) by

$$
u(x)=\sum_{k=1}^{m} u_{k}(x), \quad\left(L+d_{k}^{2}\right) u_{k}(x)=0
$$

and

$$
u(x)=\sum_{k=1}^{m / 2} \tilde{u}_{k}(x), \quad\left(L^{2}+2 b_{k} L+b_{k}^{2}+c_{k}^{2}\right) \tilde{u}_{k}(x)=0
$$

respectively. Proceeding as above, we are led to a contradiction. The proof is complete.

REMARK 1 . In the case where $\Omega=\mathbb{R}^{n}, x_{0}=0$ and $L$ is the Laplacian $\Delta$ in $\mathbb{R}^{n}$, we conclude that $\rho(x)=|x|$ and $|\nabla \rho|=1$. Then, $M[u](r)$ given by (3) reduces to the spherical mean of $u$ over $\left\{x \in \mathbb{R}^{n}:|x|=r\right\}$.

REMARK 2. In view of Lemma 1, we obtain

$$
\frac{1}{\sigma_{n} r^{n-1}} \int_{S_{r}} L^{k} u \frac{d \sigma}{|\nabla \rho|}=\left(r^{1-n} \frac{d}{d r} r^{n-1} \frac{d}{d r}\right)^{k} M[u](r),(k=1,2, \ldots, m) .
$$

Hence, we can extend the results of Naito and Yoshida [7] to the more general elliptic equation

$$
L^{m} u+a_{1} L^{m-1} u+\cdots+a_{m} u+\Phi(x, u)=f(x),
$$

where $L$ is given by (2).

REMARK 3. If $u \in C^{2 m}(\Omega)$ and $u$ satisfies (1), then $u$ is analytic in $\Omega$ (see [6, p.178]). Hence, the set of zeros of a nontrivial solution of (1) does not have interior points.

REMARK 4. Our theorem generalises a result of Górowski [5, Theorem 3]. If $L=\Delta$, our results reduce to the results of $[1,4]$ for $n=3$, and to the results of $[3,9]$ for $n \geqslant 2$.

\section{REFERENCES}

[1] F. Barański, 'The mean value theorem and oscillatory properties of certain elliptic equations in three dimensional space', Comment. Math. Prace Mat. 19 (1976), 13-14.

[2] E.A. Coddington and N. Levinson, Theory of ordinary differential equations (McGraw-Hill, New York, 1955).

[3] I.G. Dobrotvor, 'Oscillation properties of the solutions of equations with a polyharmonic operator in the space $E^{n}$, Ukrainian Math. J. 36 (1984), 230-232. 
[4] V.I. Gorbaichuk and I.G. Dobrotvor, 'Conditions for the oscillation of solutions of a class of elliptic equations of high orders with constant coefficients', Ukrainian Math. J. 32 (1980), 385-391.

[5] J. Górowski, 'The mean value theorem and oscillatory behaviour for solutions of certain elliptic equations', Math. Nachr. 122 (1985), 259-265.

[6] L. Hörmander, Linear partial differential operators (Springer-Verlag, Berlin, 1963).

[7] M. Naito and N. Yoshida, 'Oscillation criteria for a class of higher order elliptic equations', Math. Rep. Toyama Univ. 12 (1989), 29-40.

[8] N.M. Suleímanov, 'On the behavior of solutions of second order nonlinear elliptic equations with linear principal part', Soviet Math. Dokl. 20 (1979), 815-819.

[9] E. Wachnicki, 'On the oscillatory properties of solutions of certain elliptic equations', Comment. Math. Prace Mat. 19 (1976), 165-169.

[10] E. Wachnicki, 'On boundary value problems for some partial differential equations of higher order', Comment. Math. Prace Mat. 20 (1977), 215-233.

[11] B.L. van der Waerden, Algebra I (Springer-Verlag, Berlin, 1960).

[12] N. Yoshida, 'Oscillation properties of solutions of second order elliptic equations', SIAM J. Math. Anal. 14 (1983), 709-718.

Department of Mathematics

Faculty of Science

Toyama University

Toyama 930

Japan 